\title{
Syndrome D’aniridie Associé À La Dermatite Atopique: À Propos D’un Cas
}

\author{
Abba Kaka H.Y \\ Service ophtalmologique Hôpital National de Niamey \\ Salissou $L$ \\ Service de Dermatologie Hôpital National de Niamey \\ Amza A \\ Service d’ophtalmologie hôpital Lamordé \\ Daou $M$
}

Service de médecine interne Hôpital National de Niamey

doi: 10.19044/esj.2016.v12n6p97 URL:http://dx.doi.org/10.19044/esj.2016.v12n6p97

\begin{abstract}
Aniridia syndrome is a genetic anomaly affecting all ocular structures; it is transmitted by an autosomal dominant mode. In its isolated form aniridia is characterized by a hypoplasia of the iris frequently associated with other ocular anomalies. It the syndromic form it is associated to other systemic abnormalities. Authors are here reporting a case of aniridia associating: a corneal pannus, total aniridia, lens ectopia, and cataract found in a 14 years old girl. She also presented an atopic background with a positive family history of atopia. She is issued from a first degree consanguineous marriage. The management was multidisciplinary. In ophthalmology she underwent an intra-capsular extraction of the lens in both eyes with no intra-ocular lens implantation. Dermatological management was treatment of cuteanous lesions with emollients, corticoids and antihistamines drugs and ointments.
\end{abstract}

Keywords: Aniridia syndrome, atopia, Niger

\section{Résumé}

Le syndrome d'aniridie est une anomalie génétique affectant toutes les structures de l'œil, qui se transmet selon un mode autosomique dominant. L'aniridie est caractérisée dans sa forme isolée par une hypoplasie de l'iris fréquemment associée à de multiples anomalies oculaires et dans sa forme syndromique elle est associée à d'autres anomalies du système. Les auteurs rapportent le cas d'une jeune patiente de 14 ans présentant une aniridie associant: un pannus cornéen, une aniridie totale, une ectopie cristallinienne 
et une cataracte sur fond d'atopie. Cette patiente est issue d'un mariage consanguin au premier degré avec un antécédent familial d'atopie. La prise en charge fut pluridisciplinaire avec sur le plan ophtalmologique une extraction intra-capsulaire simple sans implant bilatérale. La prise en charge dermatologique des lésions lichenoides cutanées ont été par des émollients, des corticoïdes et des antihistaminiques per os et en application cutanée.

Mots-clés: Syndrome d’aniridie, atopie, Niger

\section{Introduction}

Le syndrome d'aniridie est une anomalie génétique affectant toutes les structures de l'œil (cornée, chambre antérieure, cristallin, rétine et nerf optique). Elle est due à une mutation sur le gène PAX6 sur le bras13 du chromosome 11 . Son incidence mondiale annuelle est estimée entre 1 sur 64,000 et 1 sur 96,000 naissances. Un tiers des cas est sporadique les deux tiers sont familiaux. La transmission se fait dans la majorité des cas selon le mode autosomique dominant avec une pénétrance quasi complète mais d'expression variable [1]. Elle est caractérisée dans sa forme isolée par une hypoplasie de l'iris fréquemment associée à de multiple anomalies oculaires tels que le pannus cornéen et la kératopathie, un nystagmus, une cataracte, une ectopie du cristallin, une hypoplasie fovéale, une dysfonction rétinienne, et une hypoplasie du nerf optique. Dans sa forme syndromique l'aniridie est associée à d'autres anomalies du système comme vues dans le syndrome de Miller (aniridie + tumeur de wilm) et le syndrome de Gillespie (association aniridie, ataxie cérébelleuse et un retard mental). La dermatite atopique est un eczéma constitutionnel, survenant sur un terrain génétiquement prédisposé. L’association de l'aniridie à la dermatite atopique est rare.

Nous rapportons le cas d'une jeune patiente de 14 ans, présentant un tableau syndromique d'aniridie associé à une dermatite atopique.

\section{Observation}

Mlle A.D. 14 ans, issue d'un mariage consanguin au premier degré a été reçue pour la première fois en consultation ophtalmologique pour une baisse de l'acuité visuelle progressive et bilatérale évoluant depuis l'âge de 6 ans. L'interrogatoire notait un antécédent personnel d'asthme, d'allergie alimentaire (œuf, lait, arachide), et un antécédent familial d'asthme (mère et frère). Ces symptômes sont exacerbés par une exposition prolongée au soleil. L'examen clinique a découvert une peau xérodermique associée à des squames pytiriasiformes et une hyperchromie sur le front et les extrémités (figure1: a et b). L'examen du pourtour oculaire notait un double pli palpébral au niveau des paupières inférieures ou signe de Deni Morgan 
(figure2). L’oculomotricité était normale. L’acuité visuelle était limitée à la perception lumineuse aux deux yeux.

L'examen à la lampe à fente bilatérale et comparatif a montré: un pannus cornéen supérieur sur 360 degrés, une chambre antérieure profonde et calme, une aniridie totale, une ectopie cristallinienne en supérieure, une cataracte totale, des fibres zonulaires visibles en inférieur, une condensation vitréenne et un fond'œil vu flou (figures 3 et 4). Le tonus oculaire était à 8 $\mathrm{mm} \mathrm{Hg}$ au niveau des deux yeux. Il y avait un reliquat de tissu irien à la gonioscopie. L'échographie oculaire a montré une condensation vitréenne importante et des rétines à plat.

La cataracte chez cette patiente était hyper mûre et l'ectopie était uniquement en supérieur

Les examens sanguins ont relevé une vitesse de sédimentation élevée à 120mm à la première heure, un taux sérique IgE total à 810 UI/L (n<150 UI/L). Le prick test a révélé une positivité aux crustacées, à l'arachide, à l'œuf, aux acariens.

La prise en charge chez cette patiente fut pluridisciplinaire. Sur le plan ophtalmologique elle a subi une extraction intra-capsulaire des deux cristallins, la pose d'un implant a fixation sclérale n’a pas été jugée bénéfique à cause de la condensation vitréenne importante et un manque de possibilité de vitrectomie. L'acuité postopératoire est restée stationnaire (perception lumineuse), aucune correction optique n’a été bénéfique pour cette patiente.

Le bilan dermatologique a confirmé la dermatite atopique selon les critères diagnostiques de Hanifin et Rajka, et les lésions lichenoides cutanées ont été traitées par des émollients, des corticoïdes et des antihistaminiques per os et en application cutanée. La consultation pédiatrique n’a retrouvé aucune autre anomalie systémique.

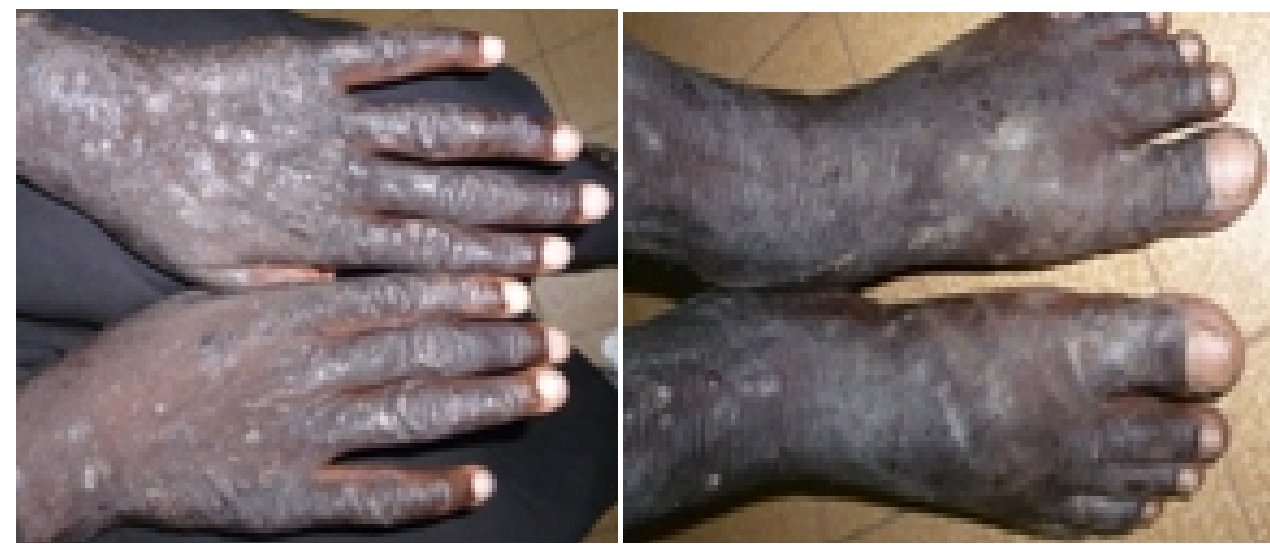

Figure 1(a et b): Lichénification des faces dorsales des pieds et des mains. 


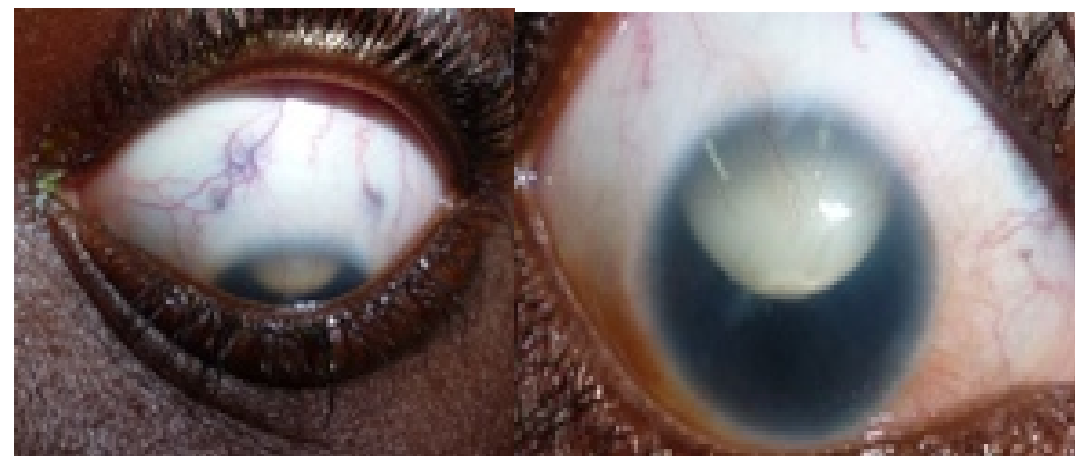

Figure 2: double plis palpébral

Figure 3: pannus, ectopie, aniridie et cataracte. (Signe de Deni Morgan).

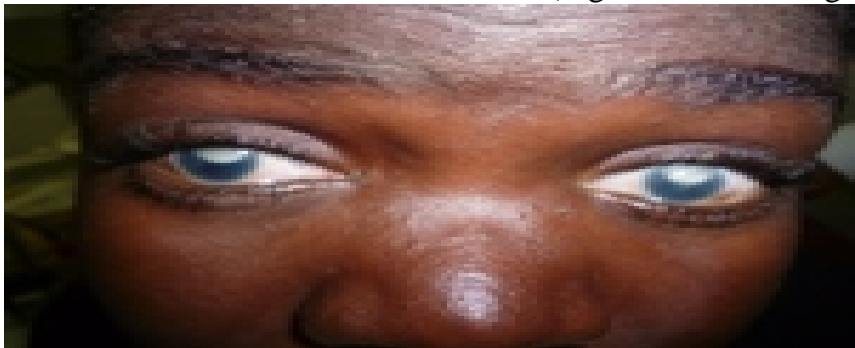

Figure 4: atteinte cutanée et oculaire bilatérale et symétrique

\section{Commentaires}

L’aniridie isolée est familiale dans les deux tiers des cas, alors que l'aniridie associée à d'autres atteintes extra oculaires est essentiellement sporadique. Dans ces cas sporadiques la consanguinité est peut être impliquée selon Angno et al. [1]. Dans les deux cas de figure, l'atteinte oculaire est en général bilatérale et les patients affectés ont un grand handicap visuel. Dans notre observation les signes oculaires associés à l'aniridie sont: le pannus cornéen, l'ectopie du cristallin et la cataracte.

Les anomalies cornéennes peuvent apparaître très précocement dans l'aniridie, avant même l'âge de 2 ans. Elles sont présentes dans 20\% des cas, toutefois ces changements de surface oculaire peuvent être observés chez presque $90 \%$ des patients [4]. Il s'agit d'une vascularisation (pannus) radiaire périphérique et superficielle associée à des opacités grisâtres dont la progression vers le centre compromet la vison. La pathogénie de cette atteinte cornéenne reste obscure, mais elle est liée à une dysgénésie iridocornéenne et serait en rapport avec une déficience en cellules épithéliales basales ou germinatives, entrainant l'envahissement de l'épithélium cornéen par l'épithélium conjonctival [2, 3]. Notre patiente présente un pannus cornéen radiaire sur $360{ }^{0}$ plus prononcé en supérieur. Ulla et al ont retrouvé $80 \%$ de cas de kératopathie chez leurs patients [5].

L'aniridie est associée à une ectopie cristallinienne dans $56 \%$ des cas [1], ceci est probablement lié à une altération moléculaire de la zonule [2-4]. 
Ndoye et al ont trouvé un cas d'ectopie sur les cinq cas étudiés [3]. La cataracte se développe chez 50 à $85 \%$ des patients présentant une aniridie [1, 8] et pose des problèmes de prise en charge chirurgicale notamment le mode de correction de l'aphaquie. Dans leur étude sur 131 patients ayant une aniridie congénitale Singh et al. ont conclu que l'atteinte la plus fréquente était celle du cristallin et la cataracte était le signe prédominant vu chez 40,3\% des patients [13]; Park et al.ont retrouvé 82,5\% de cataracte sur 62 yeux atteints d'aniridie [14].

Les atteintes oculaires sont assez fréquentes chez les patients souffrant de dermatite atopique. Leur fréquence a varié de 25 à 50\% [8, 10, 11]. La physiopathologie de ces complications oculaires n'a pu être démontrée; toutefois certaines études immunologiques comme celles de Eiichi et al. ont souligné une élévation significative du taux des IgE et d'histamine chez ces patients et surtout ceux avec la cataracte. Cette étude a également conclu que les patients souffrant d'une dermatite atopique et ayant développé une cataracte ont la forme la plus sévère de cette pathologie [10].

Au cours de la dermatite atopique la cataracte était connue bien avant l'introduction des corticoïdes locaux ou généraux qui peuvent favoriser son apparition ou accélérer son évolution [6]. L’association de la cataracte est rapportée à des fréquences variables par certains auteurs : Uehara et al. ont rapporté 12,4\% [2], Eiichi et al. 41,4\% [8], et Brunsting 10\% [9].

La particularité de notre observation est cette association entre l'aniridie et la dermatite atopique. D’autres pathologies génétiques présentent cependant des anomalies du cristallin comme l'homocystinurie et le syndrome de Marfan. Dans l'homocystinurie qui est une encéphalopathie métabolique, les complications oculaires sont les plus constantes et le plus souvent inaugurales, l'ectopie cristallinienne est retrouvée dans $90 \%$ des cas. Cette dernière est bilatérale et inférieure dans la majorité des cas mais peut être asymétrique avec une association quasi- constante d'un retard mental [13], l'aniridie et la cataracte n’y sont pas associées. Dans le syndrome de Marfan qui est une affection génétique affectant le tissu conjonctif, l'ectopie du cristallin est une caractéristique de la maladie et atteint $60 \%$ des malades, elle est en général temporo-supérieure. Il existe aussi un risque accru de décollement de la rétine, du glaucome, et de cataracte précoce, mais pas d'aniridie associée. Les aspects caractéristiques sont les anomalies aortiques et les déformations squelettiques donnant un aspect longiligne aux patients [14].

\section{Conclusion}

L'aniridie et la dermatite atopique sont toutes des anomalies génétiques et d'association rare. L’association que nous avons décrite est-elle 
liée à la consanguinité? La découverte d’autres cas et les tests génétiques seraient d'un apport bénéfique pour une bonne compréhension d'un tel cas.

\section{References:}

Dewang A, Bhaskar J, Anita P; Congenital aniridia, Journal of Current Glaucoma Practice, 2011; 5(2):1-13.

Laghmari M, Boutimzine N, Abdelouahed A.K, Lezrek M, Bensouda N, Benharbit $\mathrm{M}$ et al. Aniridie congénitale bilatérale familiale à propos de 5 cas; J Fr. Ophtalmol, 2004; 27(4) 385-391.

Ndoye Roth. P.A, Medeiros Q, Wane M.E, Khouma A.M, Dieng M, Ndiaye P.A et al. Aniridie: à propos de cinq cas; J Fr Ophtalmol; 2005; 28(8): 845849.

Calvao-Pires P, Santos-Silva.R, Falcon-Reis F, Rocha-Sousa A Congenital aniridia: clinic, genetics, therapeutics and prognosis; International Scholarly Research Notices, 2014, 10.

Ulla E, Ruth R, Kristina T. Corneal involvement in congenital aniridia; Clin Sci 2010, 29(10):1096-1102

Morand J, Schauer P, Carsuzaa F. Eil et peau, EMC 21-030-A-05.

Uehara M, Amemiya M, Arai M. Atopic cataracts in a japanese population with special reference to factors possibly relevant to cataract formation; Dermatological 1985; 170:180-184.

Eiichi U, Ikezawa M. Systemic and local immunological features of atopic dermatitis patients with ocular complications; Br J Ophthalmol 1998; 82:8287.

Brunsting L.A, Reed W.B, Bair H.L. Occurrence of cataracts and keratoconus with atopic dermatitis; Ama Arch Derm. 1955; 72(3):237-241. Esther C, Defossez-Tribout C, Ganry O; Cene S, Tramier B, Milazzo S et al ; Ocular complications of atopic dermatitis in children; Acta, 2006; 86(6):515517.

Garrity J, Liesengang T. Ocular complications of atopic dermatitis; Can J Ophthalmol 1984; 19: 21-24.

Rais L, Wafi M, Lahbil D, Iraki M, Fekkak J, Hamdani M et al.; Complications oculaires et systémiques de l'homocystinurie A propos de cinq cas. J Fr Ophtalmol 2003; 26(10):1045-1050.

Sing B, Mohamed A , Chaurasia S , Ramappa M , Mandal A.K , Jalali S; Clinical manifestations of congenital aniridia; J Pediatr Ophthalmol of Strabismus. 2014; 51(1):59-62.

Park S.H , Park Y.G , Lee M.Y , Kim M.S. Clinical features of korean patients with congenital aniridie; Korean J Ophthalmol. 2010; 24(5):291-6. 